Prons

trobertivier Journal of Nonlinear Mathematical Physics

\title{
Discretization of Liouville type nonautonomous equations preserving integrals
}

Ismagil Habibullin, Natalya Zheltukhina

To cite this article: Ismagil Habibullin, Natalya Zheltukhina (2016) Discretization of Liouville type nonautonomous equations preserving integrals, Journal of Nonlinear Mathematical Physics 23:4, 620-642, DOI:

https://doi.org/10.1080/14029251.2016.1248159

To link to this article: https://doi.org/10.1080/14029251.2016.1248159

Published online: 04 January 2021 


\title{
Discretization of Liouville type nonautonomous equations preserving integrals
}

\author{
Ismagil Habibullin \\ Institute of Mathematics, Ufa Scientific Center, Russian Academy of Sciences, \\ Chernyshevskii Str., 112, Ufa, 450077, Russia \\ and \\ Bashkir State University, Z.Validi str. 32, Ufa, 450076, Russia \\ habibullinismagil@gmail.com \\ Natalya Zheltukhina \\ Department of Mathematics, Faculty of Science, \\ Bilkent University, 06800, Ankara, Turkey \\ natalya@fen.bilkent.edu.tr
}

Received 19 July 2016

Accepted 25 September 2016

\begin{abstract}
The problem of constructing semi-discrete integrable analogues of the Liouville type integrable PDE is discussed. We call the semi-discrete equation a discretization of the Liouville type PDE if these two equations have a common integral. For the Liouville type integrable equations from the well-known Goursat list for which the integrals of minimal order are of the order less than or equal to two we presented a list of corresponding semi-discrete versions. The list contains new examples of non-autonomous Darboux integrable chains.
\end{abstract}

Keywords: Semi-discrete chain; Darboux integrability; $x$-integral, $n$-integral; continuum limit; discretization.

2000 Mathematics Subject Classification: 35Q51, 37K60

\section{Introduction}

At the present time the problem of discretization of the integrable differential equations is actively studied. In the literature one can find various approaches and techniques used to solve this problem including the Bäcklund transformation, the Hamiltonian structure, symmetries, Lax pair, finite gap integration (see [1], [2], [5], [10], [14], [15], [17], [19]). In our previous work [7] we considered the discretization of the Liouville type partial differential equations preserving the structure of one of the integrals, and we constructed the semi-discrete analogues for some equations found by $\mathrm{E}$. Goursat [6]. However, semi-discrete analogues were not found there for nonautonomous differential equations. Moreover, in [7] we did not evaluate the continuum limit equations of the chains obtained by the discretization.

In the present paper we applied the discretization via integrals procedure to nonautonomous cases as well. We also discuss continuum limit equations for some particular semi-discrete analogues obtained via the discretization. It is verified that discretization of a given Liouville type PDE found by some formal manipulations after evaluation of the continuum limit for vanishing of the grid parameter $\varepsilon$ arrives at just the same PDE.

We consider semi-discrete chains of the form

$$
\frac{d}{d x} t(n+1, x)=f\left(x, n, t(n, x), t(n+1, x), \frac{d}{d x} t(n, x)\right),
$$


where unknown function $t=t(n, x)$ depends on discrete and continuous variables $n$ and $x$, respectively. We use the following notations throughout the paper:

$$
t_{k}=t(n+k, x), \quad k \in \mathbb{Z}, \quad t_{[m]}=\frac{d^{m}}{d x^{m}} t(n, x), \quad m \in \mathbb{N} .
$$

Denote by $D$ and $D_{x}$ the shift operator and the operator of the total derivative with respect to $x$ correspondingly:

$$
\operatorname{Dh}(n, x)=h(n+1, x), \quad D_{x} h(n, x)=\frac{d}{d x} h(n, x) .
$$

Let us recall the necessary definitions (see [8], [9] for more details).

Definition 1.1. Functions $I$ and $F$, depending on $x, n,\left\{t_{[m]}\right\}_{m=1}^{\infty},\left\{t_{k}\right\}_{k=-\infty}^{\infty}$, are called respectively $n$ - and $x$-integrals of (1.1), if $D I=I$ and $D_{x} F=0$.

Any function depending on $n$ only, is an $x$-integral, and any function, depending on $x$ only, is an $n$-integral. Such integrals are called trivial integrals. One can show that any $n$-integral $I$ does not depend on variables $t_{m}$ for $m \in \mathbb{Z} \backslash\{0\}$, and any $x$-integral $F$ does not depend on variables $t_{[m]}$ for $m \in \mathbb{N}$.

Definition 1.2. Chain (1.1) is called Darboux integrable if it admits a nontrivial $n$-integral and a nontrivial $x$-integral.

Note that the order of the $n$-integral $I=I\left(n, x, t, t_{x}, \ldots, D_{x}^{m} t\right)$ equals $m$. Starting with $I$ we can produce a new integral $H$ by setting

$$
H=H\left(x, I, D_{x} I, \ldots D_{x}^{k} I\right)
$$

Evidently its order is $k+m$. It can be proved that chain (1.1) having a nontrivial integral admits a nontrivial integral of the minimal order which plays the key role: any $n$-integral $H$ can be represented in the form (1.2) through the minimal order $n$-integral $I$.

It can be verified that it is possible to find autonomous $x$ - and $y$-integrals of minimal order for any Liouville type equation of the form $u_{x y}=f\left(u, u_{x}, u_{y}\right)$, i.e. for an equation having no explicit dependence on $x, y$. This fact is clearly illustrated by the list of equations found by E.Goursat in [6]. In the recent paper [4] the authors presented a class of discrete autonomous equations possessing both nontrivial integrals of minimal orders depending on independent discrete variables. The existence of such examples, showing that the class of discrete equations has more complicated structure, stimulated our interest to the discretization problem.

Chain (1.1) is a semi-discrete analogue of the well-studied hyperbolic type equation

$$
u_{x y}=g\left(x, y, u, u_{x}, u_{y}\right) .
$$

Definition 1.3. Functions $W\left(x, y, u, u_{x}, u_{x x}, \ldots\right)$ and $\bar{W}\left(x, y, u, u_{y}, u_{y y}, \ldots\right)$, are called respectively $y$ and $x$-integrals of (1.3), if $D_{y} W=0$ and $D_{x} \bar{W}=0$.

Definition 1.4. Equation $u_{n+1, x}=f\left(x, n, u_{n}, u_{n+1}, u_{n, x}\right)$ is called a discretization of the equation (1.3) if these two equations have a common integral $W\left(x, y, u, u_{x}, u_{x x}, \ldots\right) \approx I\left(x, n, u_{n}, u_{n, x}, u_{n, x x} \ldots\right)$. Here the relation $W \approx I$ means that $I$ is obtained from $W$ by replacing $y \rightarrow n \varepsilon, u \rightarrow u_{n}, u_{x} \rightarrow u_{n, x}$, $u_{x x} \rightarrow u_{n, x x}$ and so on. 
In [6] E.Goursat presented a list of Darboux integrable equations. We selected from the list only those equations for which the minimal order integrals have the orders no greater than 2 . The trivial case when both $x$-integral $W\left(x, y, u, u_{y}\right)$ and $y$-integral $\bar{W}\left(x, y, u, u_{x}\right)$ are of order 1 is excluded:

(I) $u_{x y}=e^{u}, \bar{W}=u_{x x}-(1 / 2) u_{x}^{2}, W=u_{y y}-(1 / 2) u_{y}^{2}$;

(II) $u_{x y}=e^{u} u_{y}, \bar{W}=u_{x}-e^{u}, W=\frac{u_{y y}}{u_{y}}-u_{y}$;

(III) $u_{x y}=e^{u} \sqrt{u_{y}^{2}-4}, \bar{W}=u_{x x}-(1 / 2) u_{x}^{2}-(1 / 2) e^{2 u}, W=\frac{u_{y y}-u_{y}^{2}+4}{\sqrt{u_{y}^{2}-4}}$;

(IV) $u_{x y}=u_{x} u_{y}\left(\frac{1}{u-x}+\frac{1}{u-y}\right), \bar{W}=\frac{u_{x x}}{u_{x}}-\frac{2 u_{x}}{u-x}+\frac{1}{u-x}, W=\frac{u_{y y}}{u_{y}}-\frac{2 u_{y}}{u-y}+\frac{1}{u-y}$;

(V) $u_{x y}=\psi(u) \beta\left(u_{x}\right) \bar{\beta}\left(u_{y}\right),(\ln \psi)^{\prime \prime}=\psi^{2}, \beta \beta^{\prime}=-u_{x}, \bar{\beta} \bar{\beta}^{\prime}=-u_{y}$,

$\bar{W}=\frac{u_{x x}}{\beta\left(u_{x}\right)}-\frac{\psi^{\prime}(u)}{\psi(u)} \beta\left(u_{x}\right), W=\frac{u_{y y}}{\bar{\beta}\left(u_{y}\right)}-\frac{\psi^{\prime}(u)}{\psi(u)} \bar{\beta}\left(u_{y}\right) ;$

(VI) $u_{x y}=\frac{\beta\left(u_{x}\right) \bar{\beta}\left(u_{y}\right)}{u}, \beta \beta^{\prime}+c \beta=-u_{x}, \bar{\beta} \bar{\beta}^{\prime}+c \bar{\beta}=-u_{y}$,

$\bar{W}=\frac{u_{x x}}{\beta}-\frac{\beta}{u}, W=\frac{u_{y y}}{\bar{\beta}}-\frac{\bar{\beta}}{u}$;

(VII) $u_{x y}=-2 \frac{\sqrt{u_{x} u_{y}}}{x+y}, \bar{W}=\frac{u_{x x}}{\sqrt{u_{x}}}+2 \frac{\sqrt{u_{x}}}{x+y}, W=\frac{u_{y y}}{\sqrt{u_{y}}}+2 \frac{\sqrt{u_{y}}}{x+y}$;

(VIII) $u_{x y}=\frac{1}{(x+y) \beta\left(u_{x}\right) \bar{\beta}\left(u_{y}\right)}, \beta^{\prime}=\beta^{3}+\beta^{2}, \bar{\beta}^{\prime}=\bar{\beta}^{3}+\bar{\beta}^{2}$,

$\bar{W}=u_{x x} \beta\left(u_{x}\right)-\frac{1}{(x+y) \beta\left(u_{x}\right)}, W=u_{y y} \bar{\beta}\left(u_{y}\right)-\frac{1}{(x+y) \bar{\beta}\left(u_{y}\right)}$.

Throughout the paper we shortly call the list of eight equations above as the Goursat list. Note that the work [6] contains also equations for which the minimal order integrals are of the order higher than two.

According to Definition 1.4 in order to discretize a Darboux integrable equation of the form (1.3) we have to solve a kind of the inverse problem: search the equation of the form (1.1) possessing the given integral.

In [7] we made a discretization of equations (1.3) preserving the structure of $y$-integrals in each of eight equations from the list (I)-(VIII). The discretization in [7], where it is supposed that $n$-integrals are functions not depending on $n$, did not provide semi-discrete equations for each function $\beta\left(t_{x}\right)$ in three cases, namely cases V, VI and VIII. Also, in cases IV and VII, where $y$-integrals depend on $x$ and $y$, the obtained in [7] semi-discrete chains did not have the corresponding continuous limit equations.

\section{Statements of the results}

In the present paper we allow $n$-integral and function $f$ explicitly depend on $x$ and $n$, and with this modification in the discretization algorithm we again study all cases I - VIII. In cases V, VI and VIII the $n$-integrals depend on functions $\beta$ that are solutions of some differential equations. Below we give semi-discrete versions of these equations in the Goursat list.

Theorem 2.1. (Case V) Semi-discrete chain $t_{1 x}=f\left(x, t, t_{1}, t_{x}\right)$ possessing a minimal order n-integral $I=\frac{t_{x x}}{\beta\left(t_{x}, n\right)}+\frac{\psi^{\prime}(t, n)}{\psi(t, n)} \beta\left(t_{x}, n\right)$, where $(\ln \psi)^{\prime \prime}=\psi^{2}$ and $\beta^{\prime}\left(t_{x}, n\right) \beta\left(t_{x}, n\right)=-t_{x}$ is

$$
t_{1 x}=\lambda\left(t, t_{1}, n\right) t_{x}+\mu\left(t, t_{1}, n\right) \beta\left(t_{x}, n\right)
$$

with $\lambda$ and $\mu$ satisfying the equations

$$
\lambda^{2}+\mu^{2}=v(n), \quad \lambda_{t_{1}}-\frac{\psi^{\prime}\left(t_{1}, n+1\right)}{\psi\left(t_{1}, n+1\right)} \lambda+\frac{\psi^{\prime}(t, n)}{\psi(t, n)}=0, \quad \lambda_{t}-\frac{\psi^{\prime}(t, n)}{\psi(t, n)} \lambda+v \frac{\psi^{\prime}\left(t_{1}, n+1\right)}{\psi\left(t_{1}, n+1\right)}=0,
$$


where $v(n)$ is some constant depending on $n$ only.

This semi-discrete chain has $x$-integral $F=\psi\left(t_{1}, n+1\right) E\left(t, t_{1}, t_{2}\right)$, where $E_{t}=\frac{1}{\mu\left(t, t_{1}, n\right)}, E_{t_{2}}=$ $\frac{1}{v \mu\left(t_{1}, t_{2}, n+1\right)}$ and $E_{t_{1}}=-\frac{\lambda\left(t_{1}, t_{2}, n+1\right)}{v \mu\left(t_{1}, t_{2}, n+1\right)}-\frac{\lambda\left(t, t_{1}, n\right)}{v \mu\left(t, t_{1}, n\right)}-\frac{\psi^{\prime}\left(t_{1}, n+1\right)}{\psi\left(t_{1}, n+1\right)} E$.

Note that the overdetermined systems of the differential equations for defining $\lambda$ and, respectively $E$, are compatible (see section 3 below).

Theorem 2.2. (Case VI) Semi-discrete chain $t_{1 x}=f\left(n, t, t_{1}, t_{x}\right)$ possessing a minimal order $n$ integral $I=\frac{t_{x x}}{\beta\left(t_{x}, n\right)}-\frac{\beta\left(t_{x}, n\right)}{t}$, where $\beta^{\prime}\left(t_{x}, n\right) \beta\left(t_{x}, n\right)+C \beta\left(t_{x}, n\right)=-t_{x}$ is

$$
t_{1 x}=\lambda\left(t, t_{1}, n\right) t_{x}+\mu\left(t, t_{1}, n\right) \beta\left(t_{x}, n\right)
$$

with

$$
\left\{\begin{array}{l}
\lambda_{t}=\frac{\mu^{2}+\lambda^{2}-C \lambda \mu}{t_{1}}-\frac{\lambda}{t}, \\
\lambda_{t_{1}}=\frac{C \mu-\lambda}{t_{1}}+\frac{1}{t}
\end{array}\right.
$$

where

$$
\left\{\begin{array}{lll}
(B \lambda-\mu)^{-B^{2}}(\lambda-B \mu)=v(n), \quad B=\frac{C-\sqrt{C^{2}-4}}{{ }^{2}}, & \text { if } & C^{2}>4, \\
\ln \left(\lambda^{2}-C \lambda \mu+\mu^{2}\right)-\frac{2 C}{\sqrt{4-C^{2}}} \arctan \frac{2 \lambda-C \mu}{\mu \sqrt{4-C^{2}}}=v(n), & \text { if } & C^{2}<4, \\
\ln (\lambda-\mu)+\frac{\mu}{\lambda-\mu}=v(n), & \text { if } & C=2, \\
\ln (\lambda+\mu)-\frac{\mu}{\lambda+\mu}=v(n), & \text { if } & C=-2,
\end{array}\right.
$$

and $v(n)$ is some constant depending on $n$ only.

This semi-discrete chain has $x$-integral $F=\frac{1}{t_{1}} E\left(t, t_{1}, t_{2}\right)$, where $E_{t_{2}}=\frac{1}{\mu\left(t_{1}, t_{2}, n+1\right)}$,

$E_{t}=\frac{\mu^{2}\left(t, t_{1}, n\right)+\lambda^{2}\left(t, t_{1}, n\right)-C \lambda\left(t, t_{1}, n\right) \mu\left(t, t_{1}, n\right)}{\mu\left(t, t_{1}, n\right)}$ and $E_{t_{1}}=-\frac{\lambda\left(t_{1}, t_{2}, n+1\right)}{\mu\left(t_{1}, t_{2}, n+1\right)}-\frac{\lambda\left(t, t_{1}, n\right)}{\mu\left(t, t_{1}, n\right)}+C+\frac{1}{t_{1}} E$.

Theorem 2.3. (Case VIII) Semi-discrete chain $t_{1 x}=f\left(x, n, t, t_{1}, t_{x}\right)$ possessing a minimal order $n$ integral $I=\beta\left(t_{x}, n\right) t_{x x}-\frac{1}{(x+\alpha(n)) \beta\left(t_{x}, n\right)}$, where $\beta^{\prime}\left(t_{x}, n\right)=\beta^{3}\left(t_{x}, n\right)+\beta^{2}\left(t_{x}, n\right)$ and $\alpha(n)$ is some constant depending on $n$ only, is

$$
t_{1 x}=\frac{1-K}{\beta\left(t_{x}, n\right)}+t_{x}+(-K+\ln K)
$$

with function $K\left(x, n, t, t_{1}\right)$ satisfying the following system of equations

$$
\left\{\begin{array}{l}
K_{t}+K_{t_{1}}=0 \\
K_{t_{1}}=\frac{K}{K-1}\left\{\frac{K}{x+\alpha(n+1)}-\frac{1}{x+\alpha(n)}\right\} \\
K_{x}=\frac{K}{K-1}\left\{\frac{K}{x+\alpha(n+1)}-\frac{1}{x+\alpha(n)}\right\}(K-\ln K)-\frac{(K-1) K}{x+\alpha(n+1)} .
\end{array}\right.
$$

This semi-discrete chain has $x$-integral $F=\frac{1}{x+\alpha(n+1)} E\left(x, t, t_{1}, t_{2}\right)$, where $E_{x}=\frac{K(1-\ln K)}{1-K}-\frac{1-\ln K_{1}}{1-K_{1}}+$ $\frac{1}{x+\alpha(n+1)} E, E_{\tau_{1}}=\frac{K}{1-K}, E_{\tau_{2}}=-\frac{1}{1-K_{1}}$ with $\tau_{1}=t_{1}-t$ and $\tau_{2}=t_{2}-t_{1}$.

Let us now present one particular case described in Theorem 2.1 corresponding to $\beta\left(t_{x}\right)=\sqrt{1-t_{x}^{2}}$ and $\psi(t)=-\frac{1}{t}$. 
Example 2.1. Semi-discrete chain

$$
t_{1 x}=\frac{t_{1}^{2}+v(n) t^{2}}{2 t t_{1}} t_{x}+i \frac{v(n) t^{2}-t_{1}^{2}}{2 t t_{1}} \sqrt{1-t_{x}^{2}}
$$

has $n$-integral $I=\frac{t_{x x}}{\sqrt{1-t_{x}^{2}}}-\frac{\sqrt{1-t_{x}^{2}}}{t}$ and $x$-integral $F=\frac{v(n) t_{1}^{2}-t_{2}^{2}}{v(n) t^{2}-t_{1}^{2}}$ for any constant $v(n)$ depending on $n$ only. If in (2.3) one substitutes $u$ and $u+\varepsilon e^{\gamma\left(u_{y}\right)}$ with $\gamma^{\prime}=1 / \beta$, instead of $t$ and $t_{1}$ correspondingly, and let $\varepsilon$ approach 0 , continuous Liouville equation analogue $u_{x y}=\frac{\beta\left(u_{x}\right) \beta\left(u_{y}\right)}{u}$ would be obtained.

In cases IV and VII the $y$-integrals depend on the variables $x$ and $y$. We consider these special nonautonomous cases, allowing explicit $n$-dependence of $n$-integral and of the function $f$, and obtain some new semi-discrete chains.

Theorem 2.4. (Cases IV and VII) (a) Semi-discrete equation (1.1) possessing an n-integral $I=\frac{t_{x x}}{t_{x}}-\frac{2 t_{x}}{t-x}+\frac{1}{t-x}$ is

$$
t_{1 x}=\frac{\left(1+t_{1} M(n)\right)\left(t_{1}-x\right)}{(1+t M(n))(t-x)} t_{x}
$$

where $M(n)$ is an arbitrary function of $n$. Function $F=\frac{\left(1+t_{2} M(n+1)\right)\left(t_{1}-t\right)}{(1+t M(n))\left(t_{1}-t_{2}\right)}$ is an $x$-integral of (2.4).

(b) Semi-discrete equation (1.1) possessing an n-integral $I=\frac{t_{x x}}{\sqrt{t_{x}}}+\frac{2 \sqrt{t_{x}}}{x+\varepsilon n}$ is

$$
t_{1 x}=\left(\sqrt{t_{x}}+\alpha\right)^{2}, \alpha=\sqrt{\frac{\varepsilon\left(t_{1}-t\right)}{(x+\varepsilon n)(x+\varepsilon(n+1))}} .
$$

Function $F=(x+\varepsilon n) \alpha-(x+\varepsilon(n+2)) D \alpha$ is an $x$-integral of (2.5).

Theorem 2.5. (Cases I-IV and VII) Below we display continuum limit equations and $x$-integrals for semi-discrete equations obtained by discretization of the continuous equations from the Goursat list. 


\begin{tabular}{|c|c|c|}
\hline $\begin{array}{l}\text { Semi-discrete equation } \\
\text { and its } x \text {-integral } F\end{array}$ & $\begin{array}{l}\text { Continuum limit equations } \\
\text { and } x \text {-integrals } \tilde{F}\end{array}$ & \\
\hline $\begin{array}{l}t_{1 x}=t_{x}+C e^{(1 / 2)\left(t+t_{1}\right)}, C=\varepsilon \\
F=e^{\left(t_{1}-t\right) / 2}+e^{\left(t_{1}-t_{2}\right) / 2}\end{array}$ & $\begin{array}{l}u_{x y}=e^{u} \\
\lim _{\varepsilon \rightarrow 0} 2 \varepsilon^{-2}(2-F)=u_{y y}-(1 / 2) u_{y}^{2}=\tilde{F}\end{array}$ & $A$ \\
\hline $\begin{array}{l}t_{1 x}=t_{x}-e^{t}+e^{t_{1}} \\
F=\left(e^{t}-e^{t_{2}}\right)\left(e^{t_{1}}-e^{t_{3}}\right)\left(e^{t}-e^{t_{3}}\right)^{-1}\left(e^{t_{1}}-e^{t_{2}}\right)^{-1}\end{array}$ & $\begin{array}{l}u_{x y}=e^{u} u_{y} \\
\lim _{\varepsilon \rightarrow 0} \frac{12}{\varepsilon^{2}}(1-F)=-2 \tilde{F}_{y}+\tilde{F}^{2} \\
\tilde{F}=\frac{u_{y y}}{u_{y}}-u_{y}\end{array}$ & $B$ \\
\hline $\begin{array}{l}t_{1 x}=K\left(t, t_{1}\right) t_{x}, K=1+\varepsilon e^{t_{1}} \\
F=e^{t-t_{1}}+\varepsilon e^{t}\end{array}$ & $\begin{array}{l}u_{x y}=e^{u} u_{x} \\
\lim _{\varepsilon \rightarrow 0} \varepsilon^{-1}(1-F)=u_{y}-e^{u}=\tilde{F}\end{array}$ & $C$ \\
\hline $\begin{array}{l}t_{1 x}=t_{x}+\sqrt{e^{2 t}+R e^{t+t_{1}}+e^{2 t_{1}}}, R=-2-4 \varepsilon^{2} \\
F=\operatorname{arcsinh}\left(a e^{t_{1}-t_{2}}+b\right)+\operatorname{arcsinh}\left(a e^{t_{1}-t}+b\right) \\
a=\left(-4 \varepsilon^{4}-4 \varepsilon^{2}\right)^{-1 / 2}, b=-\left(1+2 \varepsilon^{2}\right) a\end{array}$ & $\begin{array}{l}u_{x y}=e^{u} \sqrt{u_{y}^{2}-4} \\
\lim _{\varepsilon \rightarrow 0} \varepsilon^{-1}(-F+4 \ln 2)=\frac{u_{y y}-2 u_{y}^{2}+4}{\sqrt{u_{y}^{2}-4}}=\tilde{F}\end{array}$ & $D$ \\
\hline $\begin{aligned} t_{1 x}= & \sqrt{R^{2} e^{2\left(t+t_{1}\right)}+2 R e^{t+t_{1}}} \sqrt{t_{x}^{2}-4}+ \\
& \left(1+R e^{t+t_{1}}\right) t_{x}, R=2^{-1} \varepsilon^{2} \\
F= & \sqrt{R e^{2 t_{1}}+2 e^{t_{1}-t}}+\sqrt{R e^{2 t_{1}}+2 e^{t_{1}-t_{2}}}\end{aligned}$ & $\lim _{\varepsilon \rightarrow 0} \frac{1}{\varepsilon^{2}}(4-\sqrt{2} F)=u_{y y}-\frac{1}{2}\left(u_{y}^{2}+e^{2 u}\right)$ & $E$ \\
\hline $\begin{array}{l}t_{1 x}=\frac{\left(1+t_{1} M(n)\right)\left(t_{1}-x\right)}{(1+t M(n))(t-x)} t_{x}, M=-\frac{1}{\varepsilon n} \\
F=\frac{\left(1+t_{2} M(n+1)\right)\left(t_{1}-t\right)}{(1+t M(n))\left(t_{1}-t_{2}\right)}\end{array}$ & $\begin{array}{l}u_{x y}=u_{x} u_{y}\left(\frac{1}{u-x}+\frac{1}{u-y}\right) \\
\lim _{\varepsilon \rightarrow 0} \frac{1}{\varepsilon}\left(\left(1+n^{-1}\right) F+1\right)=\frac{1-2 u_{y}}{u-y}+\frac{u_{y y}}{u_{y}}\end{array}$ & $F$ \\
\hline $\begin{array}{l}t_{1 x}=\left(\sqrt{t_{x}}+\alpha\right)^{2}, \alpha=\sqrt{\frac{\varepsilon\left(t_{1}-t\right)}{(x+\varepsilon n)(x+\varepsilon(n+1))}} \\
F=(x+\varepsilon n) \alpha-(x+\varepsilon(n+2)) D \alpha\end{array}$ & $\begin{array}{l}u_{x y}=2 \frac{\sqrt{u_{x} u_{y}}}{x+y} \\
\lim _{\varepsilon \rightarrow 0} \frac{-F}{\varepsilon^{2}}=\frac{\sqrt{u_{y}}}{x+y}+\frac{1}{2} \frac{u_{y y}}{\sqrt{u_{y}}}\end{array}$ & $G$ \\
\hline
\end{tabular}

In the present paper we concentrate mainly on the "discretization" i.e. on the evaluation of the discrete versions preserving the structure of the integrals. The inverse operation is also meaningful. According to Definition 1.4 we can look for PDE of the form (1.3) starting with the known integral of a Darboux integrable chain (1.1). Another way to find the continuous counterpart is connected with the evaluating the continuum limit. Remark that these two methods give one and the same answer. Let us give an illustrative example.

Remark 2.1. Let us find all equations $t_{x y}=f\left(x, y, t, t_{x}, t_{y}\right)$ possessing a $y$-integral $I=t_{x x}-(1 / 2) t_{x}^{2}$, that is, we are looking for a continuous analogue of semi-discrete chain $t_{1 x}=t_{x}+C e^{(1 / 2)\left(t+t_{1}\right)}$ (case (A)) preserving the structure of its $n$-integral. Equality $D_{y} I=0$ becomes $t_{x x y}-t_{x} t_{x y}=0$. From the equation searched $t_{x y}=f\left(x, y, t, t_{x}, t_{y}\right)$ we obtain $t_{x x y}=f_{x}+f_{t} t_{x}+f_{t_{x}} t_{x x}+f_{t_{y}} f$. Therefore,

$$
f_{x}+f_{t} t_{x}+f_{t_{x}} t_{x x}+f_{t_{y}} f-t_{x} f=0 .
$$


Evidently, the coefficient before $t_{x x}$ in (2.6) vanishes, that is $f_{t_{x}}=0$. Now collection of the coefficients before $t_{x}$ in (2.6) gives $f_{t}-f=0$, or $f=A\left(x, y, t_{y}\right) e^{t}$. We substitute the expression $f=A\left(x, y, t_{y}\right) e^{t}$ into (2.6) and get $A_{x} e^{t}+A_{t_{y}} e^{2 t}=0$ which immediately implies $A_{x}=A_{t_{y}}=0$. Therefore, the equation searched is of the form $t_{x y}=A(y) e^{t}$ which coincides with the Liouville equation up to a point transformation $y \rightarrow \tilde{y}=\int_{0}^{y} A(\theta) d \theta$. It is remarkable that usual continuum limit with small $\varepsilon=C>0$ approaching zero gives the same answer: equation $\left(t_{1 x}-t_{x}\right) / \varepsilon=e^{(1 / 2)\left(t+t_{1}\right)}$ becomes the Liouville equation.

Remark convinces that the problem of evaluating the PDE by its known integral is trivially solved. For the semi-discrete chain it is not the case. The matter is that in this case instead of the differential relation $D_{y} W=0$ we have a functional equation $D I=I$.

It is widely known that integrable discretization is closely connected with the Bäcklund transformation. We discuss this connection in section 8. It is shown that some of the discrete models coincide with the Bäcklund transformation for the continuous counterparts, while the others do not.

We prove Theorems $2.1-2.4$ in sections $3-6$, and present the proof of Theorem 2.5 in two special cases $F$ and $G$ in section 7. Other cases from Theorem 2.5 can be proved in a similar way.

\section{Proof of Theorem 2.1}

Discretization: Let us find all chains $t_{1 x}=f\left(x, n, t, t_{1}, t_{x}\right)$ with $n$-integral $I=\frac{t_{x x}}{\beta\left(t_{x}, n\right)}+\frac{\psi^{\prime}(t, n)}{\psi(t, n)} \beta\left(t_{x}, n\right)$, where

$$
(\ln \psi)^{\prime \prime}=\psi^{2}, \quad \beta^{\prime}\left(t_{x}, n\right)=-\frac{t_{x}}{\beta\left(t_{x}, n\right)}, \quad \beta^{\prime}(f, n+1)=-\frac{f}{\beta(f, n+1)} .
$$

$D I=I$ implies

$$
\frac{f_{x}+f_{t} t_{x}+f_{t_{1}} f+f_{t_{x}} t_{x x}}{\beta(f, n+1)}+\frac{\psi^{\prime}\left(t_{1}, n+1\right)}{\psi\left(t_{1}, n+1\right)} \beta(f, n+1)=\frac{t_{x x}}{\beta\left(t_{x}, n\right)}+\frac{\psi^{\prime}(t, n)}{\psi(t, n)} \beta\left(t_{x}, n\right) .
$$

We compare the coefficients before $t_{x x}$ and get

$$
\frac{f_{t_{x}}}{\beta(f, n+1)}=\frac{1}{\beta\left(t_{x}, n\right)}
$$

or

$$
\gamma(f, n+1)=\gamma\left(t_{x}, n\right)+A\left(x, n, t, t_{1}\right), \quad \text { where } \quad \gamma^{\prime}\left(t_{x}, n\right)=\frac{1}{\beta\left(t_{x}, n\right)} .
$$

We have, $\gamma^{\prime}(f, n+1) f_{t_{1}}=A_{t_{1}}$, or $f_{t_{1}}=A_{t_{1}} \beta(f, n+1)$. Similarly, $f_{t}=A_{t} \beta(f, n+1)$ and $f_{x}=$ $A_{x} \beta(f, n+1)$. Substitute these expressions for $f_{x}, f_{t}$ and $f_{t_{1}}$ into (3.2) and get

$$
A_{x}+t_{x} A_{t}+A_{t_{1}} f+r_{1} \beta(f, n+1)=r \beta\left(t_{x}, n\right),
$$

where

$$
r=\frac{\psi^{\prime}(t, n)}{\psi(t, n)}, \quad r_{1}=\frac{\psi^{\prime}\left(t_{1}, n+1\right)}{\psi\left(t_{1}, n+1\right)} .
$$

Differentiate with respect to $t_{x}$ equality (3.4), use (3.3) and (3.1), and get

$$
A_{t} \beta\left(t_{x}, n\right)+A_{t_{1}} \beta(f, n+1)-r_{1} f=-r t_{x} .
$$


Differentiate with respect to $t_{x}$ equality (3.6), use (3.3) and (3.1), and obtain

$$
t_{x} A_{t}+A_{t_{1}} f+r_{1} \beta(f, n+1)=r \beta\left(t_{x}, n\right) .
$$

One can see from (3.4) and (3.7) that $A_{x}=0$. We express $\beta(f, n+1)$ from (3.7), substitute it into (3.6) and get

$$
f=\lambda t_{x}+\mu \beta\left(t_{x}, n\right)
$$

where

$$
\lambda=\frac{r r_{1}-A_{t} A_{t_{1}}}{r_{1}^{2}+A_{t_{1}}^{2}}, \quad \mu=\frac{r_{1} A_{t}+r A_{t_{1}}}{r_{1}^{2}+A_{t_{1}}^{2}}
$$

Note that $f_{t_{x}}=\lambda+\mu \beta^{\prime}\left(t_{x}, n\right)=\lambda-\mu \frac{t_{x}}{\beta\left(t_{x}, n\right)}$ by (3.8) and (3.1). On the other hand, $f_{t_{x}}=\frac{\beta(f, n+1)}{\beta\left(t_{x}, n\right)}$, by (3.3). Hence,

$$
\beta(f, n+1)=-\mu t_{x}+\lambda \beta\left(t_{x}, n\right) .
$$

It follows from (3.1) that

$$
\beta^{2}\left(t_{x}, n\right)=-t_{x}^{2}+C(n), \quad \beta^{2}(f, n+1)=-f^{2}+C(n+1),
$$

where $C(n)$ and $C(n+1)$ are some constants. Since

$$
\begin{gathered}
f^{2}=\lambda^{2} t_{x}^{2}+2 \lambda \mu t_{x} \beta\left(t_{x}, n\right)+\mu^{2} \beta^{2}\left(t_{x}, n\right), \\
\beta^{2}(f, n+1)=\lambda^{2} \beta^{2}\left(t_{x}, n\right)-2 \lambda \mu t_{x} \beta\left(t_{x}, n\right)+\mu^{2} t_{x}^{2},
\end{gathered}
$$

then

$$
f^{2}+\beta^{2}(f, n+1)=\left(\lambda^{2}+\mu^{2}\right)\left(t_{x}^{2}+\beta^{2}\left(t_{x}, n\right)\right),
$$

and, therefore, due to (3.11),

$$
\lambda^{2}+\mu^{2}=v
$$

where $v=C(n+1) / C(n)$ is some constant depending on $n$ only.

Let us show that

$$
r^{2}+A_{t}^{2}=v\left(r_{1}^{2}+A_{t_{1}}^{2}\right)
$$

Indeed,

$$
v=\lambda^{2}+\mu^{2}=\frac{r^{2} r_{1}^{2}+A_{t}^{2} A_{t_{1}}^{2}+r_{1}^{2} A_{t}^{2}+r^{2} A_{t_{1}}^{2}}{\left(r_{1}^{2}+A_{t_{1}}^{2}\right)^{2}}
$$

can be rewritten as

$$
v\left(A_{t_{1}}^{2}\right)^{2}+\left(2 v r_{1}^{2}-r^{2}-A_{t}^{2}\right) A_{t_{1}}^{2}+\left(v r_{1}^{4}-r^{2} r_{1}^{2}-r_{1}^{2} A_{t}^{2}\right)=0,
$$

that implies

$$
A_{t_{1}}^{2}=\frac{-\left(2 v r_{1}^{2}-r^{2}-A_{t}^{2}\right)+r^{2}+A_{t}^{2}}{2 v}
$$

that is equivalent to (3.13). 
We substitute expressions $f=\lambda t_{x}+\mu \beta\left(t_{x}, n\right)$ and $\beta(f, n+1)=-\mu t_{x}+\lambda \beta\left(t_{x}, n\right)$ into (3.2) and get

$$
\begin{gathered}
\lambda_{t} t_{x}^{2}+\mu_{t} \beta\left(t_{x}, n\right) t_{x}+\left(\lambda_{t_{1}} t_{x}+\mu_{t_{1}} \beta\left(t_{x}, n\right)\right)\left(\lambda t_{x}+\mu \beta\left(t_{x}, n\right)\right) \\
=\left(r \beta\left(t_{x}, n\right)-r_{1} \lambda \beta\left(t_{x}, n\right)+r_{1} \mu t_{x}\right)\left(\lambda \beta\left(t_{x}, n\right)-\mu t_{x}\right) .
\end{gathered}
$$

In the last equality we first replace $\beta^{2}\left(t_{x}, n\right)$ by $-t_{x}^{2}+C(n)$ due to (3.11), and then we compare the coefficients before linearly independent functions $t_{x}^{0}, t_{x}^{2}$ and $t_{x} \beta\left(t_{x}, n\right)$. We obtain,

$$
\begin{gathered}
\mu_{t_{1}} \mu=\lambda r-\lambda^{2} r_{1}, \\
\lambda_{t}+\lambda_{t_{1}} \lambda-\mu_{t_{1}} \mu=-\lambda r+\lambda^{2} r_{1}-\mu^{2} r_{1},
\end{gathered}
$$

and

$$
\mu_{t}+\lambda_{t_{1}} \mu+\mu_{t_{1}} \lambda=-\mu r+2 \lambda \mu r_{1}
$$

Since $\lambda^{2}+\mu^{2}=v$, then $\mu_{t_{1}} \mu+\lambda_{t_{1}} \lambda=0$, and equation (3.14) becomes

$$
\lambda_{t_{1}}-r_{1} \lambda+r=0
$$

We subtract (3.14) from (3.15), use (3.17) and (3.12), and get

$$
\lambda_{t}-r \lambda+v r_{1}=0
$$

One can check that equations (3.14)-(3.16) are satisfied if and only if equations (3.17) and (3.18) hold. Note that equations (3.17) and (3.18) are compatible, since $\lambda_{t t_{1}}=\lambda_{t_{1} t}$ is equivalent to $v\left(r_{1}^{2}-\right.$ $\left.\psi_{1}^{2}\right)=r^{2}-\psi^{2}$. The last one holds because $\left(r^{2}-\psi^{2}\right)^{\prime}=2 r r^{\prime}-2 \psi \psi^{\prime}=2 \frac{\psi^{\prime}}{\psi} \psi^{2}-2 \psi \psi^{\prime}=0$ as $r^{\prime}=\psi^{2}$ by (3.1).

One can solve the system of equations (3.17) and (3.18) and get that $\lambda=v B(t) B\left(t_{1}\right) \psi(t) \psi\left(t_{1}\right)\left(\psi^{2}(t)-r^{2}(t)+C_{1}(n)\right)-r(t) B\left(t_{1}\right) \psi\left(t_{1}\right)-v r\left(t_{1}\right) B(t) \psi(t)+C_{2}(n) \psi(t) \psi\left(t_{1}\right)$, where $B^{\prime}=1 / \psi$.

Note that equation (3.8) can be written also as

$$
\gamma(f, n+1)=\gamma\left(t_{x}, n\right)+A\left(t, t_{1}, n\right)
$$

where, due to (3.9) and (3.13), we have

$$
\gamma^{\prime}\left(t_{x}, n\right)=\frac{1}{\beta\left(t_{x}, n\right)}, \quad A_{t_{1}}^{2}=\frac{\left(r-\lambda r_{1}\right)^{2}}{v-\lambda^{2}}, \quad A_{t}^{2}=\frac{\left(v r_{1}-\lambda r\right)^{2}}{v-\lambda^{2}},
$$

and $\lambda$ satisfies (3.17) and (3.18). 
Finding $x$-integral: Now we are looking for an $x$-integral $F\left(t, t_{1}, t_{2}\right)$ of equation (3.8) satisfying (3.12), (3.17) and (3.18). Equality $D_{x} F=0$ implies

$$
F_{t} t_{x}+F_{t_{1}}\left(\lambda t_{x}+\mu \beta\left(t_{x}, n\right)\right)+F_{t_{2}}\left(\left(\lambda_{1} \lambda-\mu_{1} \mu\right) t_{x}+\left(\lambda_{1} \mu+\mu_{1} \lambda\right) \beta\left(t_{x}, n\right)\right)=0 .
$$

By comparing the coefficients before $t_{x}$ and $\beta\left(t_{x}, n\right)$ in the last equation we get the system of two equations

$$
\left\{\begin{array}{r}
F_{t}+\lambda F_{t_{1}}+\left(\lambda_{1} \lambda-\mu_{1} \mu\right) F_{t_{2}}=0 \\
\mu F_{t_{1}}+\left(\lambda_{1} \mu+\mu_{1} \lambda\right) F_{t_{2}}=0
\end{array}\right.
$$

that can be rewritten as

$$
\left\{\begin{array}{l}
F_{t}-v \frac{\mu_{1}}{\mu} F_{t_{2}}=0 \\
F_{t_{1}}+\left(\lambda_{1}+\lambda \frac{\mu_{1}}{\mu}\right) F_{t_{2}}=0
\end{array}\right.
$$

Note that $\left[\frac{\partial}{\partial t}-v \frac{\mu_{1}}{\mu} \frac{\partial}{\partial t_{2}}, \frac{\partial}{\partial t_{1}}+\left(\lambda_{1}+\lambda \frac{\mu_{1}}{\mu}\right) \frac{\partial}{\partial t_{2}}\right]=\left(\mu_{1}\left(\frac{\lambda}{\mu}\right)_{t}-v \mu\left(\frac{\lambda_{1}}{\mu_{1}}\right)_{t_{2}}+v\left(\frac{\mu_{1}}{\mu}\right)_{t_{1}}\right) \frac{\partial}{\partial t_{2}}=0$ due to (3.12), (3.17) and (3.18). Define function $E\left(t, t_{1}, t_{2}\right)$ to be such that

$$
E_{t}=\frac{1}{\mu}, \quad E_{t_{2}}=\frac{1}{v \mu_{1}}, \quad E_{t_{1}}=-\frac{\lambda_{1}}{v \mu_{1}}-\frac{\lambda}{v \mu}-r_{1} E .
$$

Such function exists since $E_{t t_{2}}=0=E_{t_{2} t}$ and $E_{t t_{1}}=E_{t_{1}}, E_{t_{1} t_{2}}=E_{t_{2} t_{1}}$ by (3.12), (3.17) and (3.18). In new variables $\tilde{t}=E\left(t, t_{1}, t_{2}\right), \tilde{t_{1}}=t_{1}, \tilde{t_{2}}=t_{2}$ system (3.20) becomes

$$
\left\{\begin{array}{l}
F_{\tilde{t}_{2}}=0, \\
F_{\tilde{t}_{1}}-\frac{\psi^{\prime}\left(\tilde{t_{1}}\right)}{\psi\left(\tilde{t}_{1}\right)} \tilde{t} F_{\tilde{t}}=0 .
\end{array},\right.
$$

that implies that $x$-integral can be taken as $F\left(t, t_{1}, t_{2}\right)=\psi\left(t_{1}\right) E\left(t, t_{1}, t_{2}\right)$, where $E$ satisfies (3.21).

\section{Proof of Theorem 2.2}

Discretization: Consider chains $t_{1 x}=f\left(n, t, t_{1}, t_{x}\right)$ with $n$-integral $I=\frac{t_{x x}}{\beta\left(t_{x}, n\right)}-\frac{\beta\left(t_{x}, n\right)}{t}$, where

$$
\beta\left(t_{x}, n\right) \beta^{\prime}\left(t_{x}, n\right)+C \beta\left(t_{x}, n\right)=-t_{x} .
$$

Equality $D I=I$ implies

$$
\frac{f_{t} t_{x}+f_{t_{1}} f+f_{t_{x}} t_{x x}}{\beta(f, n+1)}-\frac{\beta(f, n+1)}{t_{1}}=\frac{t_{x x}}{\beta\left(t_{x}, n\right)}-\frac{\beta\left(t_{x}, n\right)}{t} .
$$

By comparing the coefficients before $t_{x x}$ in (4.2) we get

$$
\frac{f_{t_{x}}}{\beta(f, n+1)}=\frac{1}{\beta\left(t_{x}, n\right)}, \quad \text { or } \quad \gamma(f, n+1)-\gamma\left(t_{x}, n\right)=A\left(t, t_{1}, n\right) \quad \text { with } \gamma^{\prime}=1 / \beta \text {. }
$$

It follows from (4.3) that $f_{t}=\beta(f, n+1) A_{t}$ and $f_{t_{1}}=\beta(f, n+1) A_{t_{1}}$. We substitute these expressions for $f_{t}$ and $f_{t_{1}}$ into (4.2) and obtain

$$
A_{t} t_{x}+A_{t_{1}} f=\frac{\beta(f, n+1)}{t_{1}}-\frac{\beta\left(t_{x}, n\right)}{t} .
$$


The next system of two equations is the results of differentiation of (4.4) with respect to $t_{x}$ consequently and usage of (4.1) and (4.3).

$$
\left\{\begin{array}{l}
\left(A_{t_{1}}+\frac{C}{t_{1}}\right) \beta(f, n+1)+\frac{1}{t_{1}} f=\frac{t_{x}}{t}+\left(\frac{C}{t}-A_{t}\right) \beta\left(t_{x}, n\right), \\
\left(C A_{t_{1}}+\frac{C^{2}-1}{t_{1}}\right) \beta(f, n+1)+\left(A_{t_{1}}+\frac{C}{t_{1}}\right) f=\left(\frac{C}{t}-A_{t}\right) t_{x}+\left(\frac{C^{2}-1}{t}-C A_{t}\right) \beta\left(t_{x}, n\right) .
\end{array}\right.
$$

This system of two linear equations with respect to $\beta(f, n+1)$ and $f$ implies that $f$ can be written as

$$
f=\lambda\left(t, t_{1}\right) t_{x}+\mu\left(t, t_{1}\right) \beta\left(t_{x}, n\right),
$$

where

$$
\lambda=\frac{t_{1}-t t_{1}^{2} A_{t} A_{t_{1}}-C t t_{1} A_{t}}{C t t_{1} A_{t_{1}}+C^{2} t+t} \quad \text { and } \quad \mu=-\frac{t t_{1} A_{t}+t_{1}^{2} A_{t_{1}}}{C t t_{1} A_{t_{1}}+C^{2} t+t} .
$$

It follows from (4.5) and (4.1) that $f_{t_{x}}=\lambda+\mu \beta^{\prime}\left(t_{x}, n\right)=\lambda-\mu\left(C+\frac{t_{x}}{\beta\left(t_{x}, n\right)}\right)$. On the other hand, by (4.3), we have $f_{t_{x}}=\beta(f, n+1) / \beta\left(t_{x}, n\right)$. Therefore,

$$
\beta(f, n+1)=-\mu t_{x}+(\lambda-C \mu) \beta\left(t_{x}, n\right) .
$$

We substitute $f=\lambda t_{x}+\mu \beta\left(t_{x}, n\right)$ and $\beta(f, n+1)=(\lambda-C \mu) \beta\left(t_{x}, n\right)-\mu t_{x}$ into (4.2) and get

$$
\begin{gathered}
\lambda_{t} t_{x}^{2}+\mu_{t} t_{x} \beta\left(t_{x}, n\right)+\lambda \lambda_{t_{1}} t_{x}^{2}+\lambda_{t_{1}} \mu \beta\left(t_{x}, n\right) t_{x}+\mu_{t_{1}} \lambda t_{x} \beta\left(t_{x}, n\right)+\mu_{t_{1}} \mu \beta^{2}\left(t_{x}, n\right) \\
=\frac{(\lambda-C \mu)^{2} \beta^{2}\left(t_{x}, n\right)}{t_{1}}-\frac{2(\lambda-C \mu) \mu t_{x} \beta\left(t_{x}, n\right)}{t_{1}}+\frac{\mu^{2} t_{x}^{2}}{t_{1}}-\frac{(\lambda-C \mu) \beta^{2}\left(t_{x}, n\right)}{t}+\frac{\mu t_{x} \beta\left(t_{x}, n\right)}{t},
\end{gathered}
$$

that implies, after comparing coefficients before linearly independent functions $t_{x}^{2}, t_{x} \beta\left(t_{x}, n\right)$ and $\beta^{2}\left(t_{x}, n\right)$, the following system of equations on $\lambda$ and $\mu$ takes place

$$
\left\{\begin{array}{l}
\lambda_{t}+\lambda \lambda_{t_{1}}=\frac{\mu^{2}}{t_{1}}, \\
\mu_{t}+\lambda_{t_{1}} \mu+\mu_{t_{1}} \lambda=\frac{2(C \mu-\lambda) \mu}{t_{1}}+\frac{\mu}{t}, \\
\mu_{t_{1}} \mu=\frac{(\lambda-C \mu)^{2}}{t_{1}}+\frac{C \mu-\lambda}{t} .
\end{array}\right.
$$

Note that the Wronskian of functions $t_{x}^{2}, t_{x} \beta\left(t_{x}, n\right)$ and $\beta^{2}\left(t_{x}, n\right)$ is equal to $2\left(t_{x} \beta^{\prime}\left(t_{x}, n\right)-\beta\left(t_{x}, n\right)\right)^{3}$. It is equal to 0 if and only if $\beta\left(t_{x}, n\right)=\frac{-C \pm \sqrt{C^{2}-4}}{2} t_{x}$ provided that function $\beta$ satisfies (4.1). In this case, due to (4.5), we would have $t_{1 x}=K\left(t, t_{1}\right) t_{x}$. Otherwise, the Wronskian is not 0 that implies that functions $t_{x}^{2}, t_{x} \beta\left(t_{x}, n\right)$ and $\beta^{2}\left(t_{x}, n\right)$ are indeed linearly independent.

Let us find the relation between $\lambda$ and $\mu$. Denote by

$$
w=\frac{\beta\left(t_{x}, n\right)}{t_{x}} .
$$

Equation (4.1) becomes

$$
\frac{w d w}{w^{2}+C w+1}=-\frac{d t_{x}}{t_{x}}
$$

We study this equation in three different cases.

$\underline{\text { Case 1) }}$ is when $C^{2}>4$ and, therefore, $w^{2}+C w+1=\left(w+\frac{C}{2}\right)^{2}-\frac{C^{2}-4}{4}$. 
Case 2) is when $C^{2}<4$ and, therefore, $w^{2}+C w+1=\left(w+\frac{C}{2}\right)^{2}+\frac{4-C^{2}}{4}$.

$\underline{\text { Case 3) }}$ is when $C^{2}=4$ and, therefore, $w^{2}+C w+1=\left(w+\frac{C}{2}\right)^{2}$.

In Case 1) the solution of (4.8) is

$$
(w+B)^{-B^{2}}\left(w+\frac{1}{B}\right) t_{x}^{1-B^{2}}=\text { Const }_{1}(n) \quad \text { with } \quad B=\frac{C-\sqrt{C^{2}-4}}{2},
$$

that can be rewritten as

$$
\left(\beta\left(t_{x}, n\right)+B t_{x}\right)^{-B^{2}}\left(B \beta\left(t_{x}, n\right)+t_{x}\right)=\text { Const }_{1} .
$$

Also,

$$
(\beta(f, n+1)+B f)^{-B^{2}}(B \beta(f, n+1)+f)=\text { Const }_{2} .
$$

We substitute (4.6) into (4.10), use (4.9), and get that in Case 1) there is the following relation between $\lambda$ and $\mu$ :

$$
(B \lambda-\mu)^{-B^{2}}(\lambda-B \mu)=v(n), \quad B=\frac{C-\sqrt{C^{2}-4}}{2} .
$$

Differentiation of (4.11) with respect to $t$ and $t_{1}$ gives the following equations

$$
\left\{\begin{array}{l}
\mu \mu_{t}=(C \mu-\lambda) \lambda_{t} \\
\mu \mu_{t_{1}}=(C \mu-\lambda) \lambda_{t_{1}}
\end{array}\right.
$$

In Case 2) the solution of (4.8) is

$$
\ln \left(w^{2} t_{x}^{2}+C w t_{x}^{2}+t_{x}^{2}\right)-\frac{2 C}{\sqrt{4-C^{2}}} \arctan \frac{2 w+C}{\sqrt{4-C^{2}}}=\text { Const }_{1},
$$

that can be rewritten as

$$
\ln \left(\beta^{2}\left(t_{x}, n\right)+C t_{x} \beta\left(t_{x}, n\right)+t_{x}^{2}\right)-\frac{2 C}{\sqrt{4-C^{2}}} \arctan \frac{2 \beta\left(t_{x}, n\right)+C t_{x}}{t_{x} \sqrt{4-C^{2}}}=\text { Const }_{1} .
$$

Also,

$$
\ln \left(\beta^{2}(f, n+1)+C f \beta(f, n+1)+f^{2}\right)-\frac{2 C}{\sqrt{4-C^{2}}} \arctan \frac{2 \beta(f, n+1)+C f}{f \sqrt{4-C^{2}}}=\text { Const }_{2} .
$$

We substitute (4.6) into (4.14), use (4.13), and get that in Case 2) there is the following relation between $\lambda$ and $\mu$ :

$$
\ln \left(\lambda^{2}-C \lambda \mu+\mu^{2}\right)-\frac{2 C}{\sqrt{4-C^{2}}} \arctan \frac{2 \lambda-C \mu}{\mu \sqrt{4-C^{2}}}=v(n) .
$$

Differentiation of (4.15) with respect to $t$ and $t_{1}$ gives (4.12).

We study Case 3) in the same way as Cases 1) and 2) and get the following relation between $\lambda$ and $\mu$ :

$$
\left\{\begin{array}{lll}
\ln (\lambda-\mu)+\frac{\mu}{\lambda-\mu}=v(n), & \text { if } & C=2, \\
\ln (\lambda+\mu)-\frac{\mu}{\lambda+\mu}=v(n), & \text { if } & C=-2 .
\end{array}\right.
$$

Differentiation of (4.16) with respect to $t$ and $t_{1}$ gives (4.12). 
In all three cases we substitute the expressions for $\mu_{t}$ and $\mu_{t_{1}}$ from (4.12) into (4.7) and have (2.1). Note that system (2.1) is compatible, i.e. $\lambda_{t t_{1}}=\lambda_{t_{1}}$, if and only if equations (4.12) hold.

Finding $x$-integral: Let us find function $F\left(t, t_{1}, t_{2}\right)$ such that $0=D_{x} F=F_{t} t_{x}+F_{t_{1}} t_{1 x}+F_{t_{2}} t_{2 x}$. Due to (4.5) and (4.6), we have $t_{1 x}=\lambda t_{x}+\mu \beta\left(t_{x}, n\right)$ and $t_{2 x}=\left(\lambda_{1} \lambda-\mu_{1} \mu\right) t_{x}+\left(\lambda_{1} \mu+\mu_{1} \lambda-\right.$ $\left.C \mu \mu_{1}\right) \beta\left(t_{x}, n\right)$, where $\lambda_{1}=D \lambda$ and $\mu_{1}=D \mu$. By comparing the coefficients in $D_{x} F=0$ before $t_{x}$ and $\beta\left(t_{x}, n\right)$ we get the following system of two equations

$$
\left\{\begin{array}{l}
F_{t}+\lambda F_{t_{1}}+\left(\lambda_{1} \lambda-\mu_{1} \mu\right) F_{t_{2}}=0 \\
\mu F_{t_{1}}+\left(\lambda_{1} \mu+\mu_{1} \lambda-C \mu \mu_{1}\right) F_{t_{2}}=0
\end{array}\right.
$$

that can be rewritten as

$$
\left\{\begin{array}{l}
\mu F_{t}+\mu_{1}\left(C \lambda \mu-\mu^{2}-\lambda^{2}\right) F_{t_{2}}=0 \\
\mu F_{t_{1}}+\left(\lambda_{1} \mu+\mu_{1} \lambda-C \mu \mu_{1}\right) F_{t_{2}}=0 .
\end{array}\right.
$$

Let $E\left(t, t_{1}, t_{2}\right)$ be such that $E_{t}=\frac{\mu^{2}+\lambda^{2}-C \lambda \mu}{\mu}, E_{t_{1}}=-\frac{\lambda_{1}}{\mu_{1}}-\frac{\lambda}{\mu}+C+\frac{1}{t_{1}} E$ and $E_{t_{2}}=\frac{1}{\mu_{1}}$. Such function $E$ exists since $E_{t t_{2}}=0=E_{t_{2} t}$ and $E_{t t_{1}}=E_{t_{1} t}, E_{t_{1} t_{2}}=E_{t_{2} t_{1}}$ provided equations (4.12) hold.

In new variables $\tilde{t}=E\left(t, t_{1}, t_{2}\right), \tilde{t_{1}}=t_{1}, \tilde{t_{2}}=t_{2}$ the system (4.17) becomes

$$
\left\{\begin{array}{l}
F_{\tilde{t}_{2}}=0 \\
\tilde{t} F_{\tilde{t}}+\tilde{t_{1}} F_{\tilde{t}_{1}}=0
\end{array}\right.
$$

One can see that $x$-integral then can be taken as $F\left(t, t_{1}, t_{2}\right)=\frac{1}{t_{1}} E\left(t, t_{1}, t_{2}\right)$.

\section{Proof of Theorem 2.3}

Discretization: Consider chains $t_{1 x}=f\left(x, n, t, t_{1}, t_{x}\right)$ with $n$-integral $I=\beta\left(t_{x}, n\right) t_{x x}-\frac{1}{(x+\alpha(n)) \beta\left(t_{x}, n\right)}$, where

$$
\beta^{\prime}\left(t_{x}, n\right)=\beta^{3}\left(t_{x}, n\right)+\beta^{2}\left(t_{x}, n\right)
$$

Denote by

$$
\beta=\beta\left(t_{x}, n\right), \quad \bar{\beta}=\beta(f, n+1), \quad \alpha=\alpha(n), \quad \alpha_{1}=\alpha(n+1) .
$$

Since $D I=I$ then

$$
\bar{\beta}\left(f_{x}+f_{t} t_{x}+f_{t_{1}} f+f_{t_{x}} t_{x x}\right)-\frac{1}{\left(x+\alpha_{1}\right) \bar{\beta}}=\beta t_{x x}-\frac{1}{(x+\alpha) \beta} .
$$

By comparing the coefficients in (5.2) before $t_{x x}$ we have

$$
\bar{\beta} f_{t_{x}}=\beta, \quad \text { or } \quad \gamma(f, n+1)-\gamma\left(t_{x}, n\right)=A\left(x, n, t, t_{1}\right) \quad \text { with } \quad \gamma^{\prime}=\beta .
$$

It follows from (5.3) that $f_{t}=A_{t} / \bar{\beta}, f_{t_{1}}=A_{t_{1}} / \bar{\beta}$ and $f_{x}=A_{x} / \bar{\beta}$. Substitute these expressions for $f_{x}$, $f_{t}, f_{t_{1}}$ into (5.2) and get

$$
A_{x}+A_{t} t_{x}+A_{t_{1}} f=\frac{1}{x+\alpha_{1}} \bar{\mu}-\frac{1}{x+\alpha} \mu
$$

where

$$
\mu=\frac{1}{\beta\left(t_{x}, n\right)} \quad \text { and } \quad \bar{\mu}=\frac{1}{\beta(f, n+1)} .
$$


Note that equation (5.1) in terms of $\mu$ can be rewritten as

$$
-\mu \mu^{\prime}=1+\mu .
$$

Therefore,

$$
\mu\left(t_{x}, n\right)-\ln \left(1+\mu\left(t_{x}, n\right)\right)+t_{x}=C_{1},
$$

where $C_{1}$ is some constant depending on $n$ only.

We differentiate (5.4) with respect to $t_{x}$, use (5.3), (5.5), (5.6) and get

$$
A_{t}+A_{t_{1}} \frac{\bar{\mu}}{\mu}=-\frac{1}{x+\alpha_{1}} \frac{1+\bar{\mu}}{\mu}+\frac{1}{x+\alpha} \frac{1+\mu}{\mu}
$$

that is equivalent to

$$
\mu(f, n+1)=\frac{(x+\alpha)^{-1}-A_{t}}{\left(x+\alpha_{1}\right)^{-1}+A_{t_{1}}} \mu\left(t_{x}, n\right)+\frac{(x+\alpha)^{-1}-\left(x+\alpha_{1}\right)^{-1}}{A_{t_{1}}+\left(x+\alpha_{1}\right)^{-1}},
$$

or $A_{t_{1}}=-\left(x+\alpha_{1}\right)^{-1}, A_{t}=(x+\alpha)^{-1}$ and $\alpha=\alpha_{1}$ (in this case $\gamma\left(t_{1 x}\right)=\gamma\left(t_{x}\right)+\left(t-t_{1}\right)(x+\alpha)^{-1}$ by (5.3)).

Differentiate (5.8) with respect to $t_{x}$, use (5.3), (5.5) and (5.6), and get

$$
A_{t_{1}}(\bar{\mu}-\mu)+\frac{1+\bar{\mu}}{x+\alpha_{1}}=\frac{1+\mu}{x+\alpha},
$$

or

$$
\mu(f, n+1)=\left(\frac{A_{t_{1}}+(x+\alpha)^{-1}}{A_{t_{1}}+\left(x+\alpha_{1}\right)^{-1}}\right) \mu\left(t_{x}, n\right)+\frac{(x+\alpha)^{-1}-\left(x+\alpha_{1}\right)^{-1}}{A_{t_{1}}+\left(x+\alpha_{1}\right)^{-1}} .
$$

By comparing the last equation with (5.9) we get

$$
A_{t}+A_{t_{1}}=0
$$

Note that, by (5.10), we have

$$
1+\bar{\mu}=\frac{A_{t_{1}}+(x+\alpha)^{-1}}{A_{t_{1}}+\left(x+\alpha_{1}\right)^{-1}}(1+\mu)
$$

It follows from (5.7) that

$$
\mu(f, n+1)-\ln (1+\mu(f, n+1))+f=C_{2} .
$$

Substitute (5.12), (5.10) into (5.13), use (5.7) and obtain

$$
f=(1-K) \mu\left(t_{x}, n\right)+t_{x}+(\ln K-K)+C_{2}-C_{1}+1, \quad \text { where } \quad K=\frac{A_{t_{1}}+(x+\alpha)^{-1}}{A_{t_{1}}+\left(x+\alpha_{1}\right)^{-1}} .
$$

Observe that

$$
K_{t}=\left(1+\frac{(x+\alpha)^{-1}-\left(x+\alpha_{1}\right)^{-1}}{A_{t_{1}}+\left(x+\alpha_{1}\right)^{-1}}\right)_{t}=\frac{\left(x+\alpha_{1}\right)^{-1}-(x+\alpha)^{-1}}{\left(A_{t_{1}}+\left(x+\alpha_{1}\right)^{-1}\right)^{2}} A_{t_{1} t}
$$




$$
=\frac{\left(x+\alpha_{1}\right)^{-1}-(x+\alpha)^{-1}}{\left(A_{t_{1}}+\left(x+\alpha_{1}\right)^{-1}\right)^{2}} A_{t t_{1}}=-\frac{\left(x+\alpha_{1}\right)^{-1}-(x+\alpha)^{-1}}{\left(A_{t_{1}}+\left(x+\alpha_{1}\right)^{-1}\right)^{2}} A_{t_{1} t_{1}}=-K_{t_{1}}
$$

by (5.11), i.e.

$$
K_{t}+K_{t_{1}}=0
$$

Substitute (5.14) into (5.2), use (5.10) and (5.15), compare the coefficients in the obtained equality before linearly independent functions $t_{x}^{0}, \mu$ and $\mu^{2}$ (the Wronskian of $t_{x}^{0}, \mu$ and $\mu^{2}$ is equal to $-2(1+\mu)^{3} \mu^{-3} \neq 0$ unless $\left.\mu=-1\right)$, and get that function $K\left(x, n, t, t_{1}\right)$ must satisfy (2.2). One can check that system (2.2) is consistent since $(K-1)^{2}(\ln K)_{t_{1} x}=(K-1)^{2}(\ln K)_{x t_{1}}$, i.e. $K_{t_{1} x}=K_{x t_{1}}$.

Finding $x$-integral: Let us find function $F\left(x, t, t_{1}, t_{2}\right)$ such that

$$
0=D_{x} F=F_{x}+F_{t} t_{x}+F_{t_{1}} t_{1 x}+F_{t_{2}} t_{2 x}
$$

Note that, due to the fact that $t_{1 x}=(1-K) \mu\left(t_{x}, n\right)+t_{x}+(-K+\ln K)$ and $\mu(f, n+1)=K \mu\left(t_{x}, n\right)+$ $(K-1)$ by (5.10), we have

$$
t_{2 x}=\left(1-K K_{1}\right) \mu\left(t_{x}, n\right)+t_{x}+\left(-1-K K_{1}+\ln \left(K K_{1}\right)\right) .
$$

Functions $t_{x}^{0}, t_{x}$ and $\mu\left(t_{x}, n\right)$ are linearly independent since their Wronskian is equal to $-(1+$ $\mu) \mu^{-3} \neq 0$ unless $\mu=-1$. We compare the coefficients before $t_{x}^{0}, t_{x}$ and $\mu\left(t_{x}, n\right)$ in $D_{x} F=0$ and get

$$
\left\{\begin{array}{l}
F_{x}+(\ln K-K) F_{t_{1}}+\left(\ln \left(K K_{1}\right)-\left(K K_{1}\right)-1\right) F_{t_{2}}=0 \\
F_{t}+F_{t_{1}}+F_{t_{2}}=0 \\
(1-K) F_{t_{1}}+\left(1-K K_{1}\right) F_{t_{2}}=0
\end{array}\right.
$$

In new variables $\tau=t, \tau_{1}=t_{1}-t$ and $\tau_{2}=t_{2}-t_{1}$ the system (5.16) can be written as

$$
\left\{\begin{array}{l}
A(F)=F_{x}+\left\{\frac{K\left(1-K_{1}\right)(1-\ln K)}{1-K}+\ln K_{1}-1\right\} F_{\tau_{2}}=0 \\
F_{\tau}=0 \\
B(F)=(1-K) F_{\tau_{1}}+K\left(1-K_{1}\right) F_{\tau_{2}}=0
\end{array}\right.
$$

One can check that the last system is closed since $[A, B]=\left(1-K^{-1}\right) K_{\tau_{1}} A+K_{x} K^{-2} B$. Note that $K=K\left(\tau_{1}\right)$ and then $K_{1}=K\left(\tau_{2}\right)$. Define function $E\left(x, t, t_{1}, t_{2}\right)$, where $E_{x}=\frac{K(1-\ln K)}{1-K}-\frac{1-\ln K_{1}}{1-K_{1}}+$ $\frac{1}{x+\alpha(n+1)} E, E_{\tau_{1}}=\frac{K}{1-K}, E_{\tau_{2}}=-\frac{1}{1-K_{1}}$. Such function exists since $E_{\tau_{1} \tau_{2}}=0=E_{\tau_{2} \tau_{1}}$ and $E_{x \tau_{1}}=E_{\tau_{1} x}$, $E_{x \tau_{2}}=E_{\tau_{2} x}$ due to (2.2) and the fact that $K_{\tau_{1}}\left(\tau_{1}\right)=K_{t_{1}}\left(t, t_{1}\right)$.

Introduce $\tau_{1}^{*}=\tau_{1}$ and $\tau_{2}^{*}=E\left(x, \tau_{1}, \tau_{2}\right)$. The first and the third equations of (5.17) become

$$
\left\{\begin{array}{l}
F_{x}+\frac{\tau_{2}^{*}}{x+\alpha_{1}} F_{\tau_{2}^{*}}=0 \\
F_{\tau_{1}^{*}}=0
\end{array}\right.
$$

that implies that $x$-integral can be taken as $F=\frac{1}{x+\alpha_{1}} E\left(x, \tau_{1}, \tau_{2}\right)$. 


\section{Proof of Theorem 2.4}

Discretization, Part(a): We consider semi-discrete equations $t_{1 x}=f\left(x, n, t, t_{1}, t_{x}\right)$ with $n$-integral

$$
I=\frac{t_{x x}}{t_{x}}-\frac{2 t_{x}}{t-x}+\frac{1}{t-x}
$$

From $D I=I$ we get

$$
\frac{f_{x}+f_{t} t_{x}+f_{t_{1}} f+f_{t_{x}} t_{x x}}{f}-\frac{2 f}{t_{1}-x}+\frac{1}{t_{1}-x}=\frac{t_{x x}}{t_{x}}-\frac{2 t_{x}}{t-x}+\frac{1}{t-x}
$$

By comparing the coefficients in (6.2) before $t_{x x}$ we obtain $f_{t_{x}} / f=1 / t_{x}$, or $f=t_{x} K$, where $K$ is some function depending on $x, n, t$ and $t_{1}$. Substitute $f=t_{x} K$ into (6.2) and find

$$
\frac{K_{x} t_{x}+K_{t} t_{x}^{2}+K_{t_{1}} K t_{x}^{2}}{K t_{x}}-\frac{2 K t_{x}}{t_{1}-x}+\frac{1}{t_{1}-x}=-\frac{2 t_{x}}{t-x}+\frac{1}{t-x}
$$

Compare the coefficients before $t_{x}$ and $t_{x}^{0}$ in (6.3) and get

$$
\begin{gathered}
\frac{K_{t}}{K}+K_{t_{1}}=\frac{2 K}{t_{1}-x}-\frac{2}{t-x} \\
\frac{K_{x}}{K}=\frac{1}{t-x}-\frac{1}{t_{1}-x}
\end{gathered}
$$

We solve (6.5) and have $K=C\left(t_{1}-x\right) /(t-x)$, where $C$ is some function depending on $n, t$ and $t_{1}$. Substitute this expression for $K$ into (6.4) and obtain

$$
\frac{C_{t}}{C}(t-x)+C_{t_{1}}\left(t_{1}-x\right)=C-1
$$

By comparing the coefficients before $x$ and $x^{0}$ in (6.6) we get the system of equations

$$
\left\{\begin{array}{l}
\frac{C_{t}}{C}+C_{t_{1}}=0, \\
\frac{C_{t}}{C} t+C_{t_{1}} t_{1}=C-1
\end{array}\right.
$$

whose solution is $C=\left(1+M(n) t_{1}\right) /(1+M(n) t)$. Thus, equation $t_{1 x}=f\left(x, n, t, t_{1}, t_{x}\right)$ possessing $n$-integral (6.1) is

$$
t_{1 x}=\frac{\left(1+M(n) t_{1}\right)\left(t_{1}-x\right)}{(1+M(n) t)(t-x)} t_{x}
$$

where $M(n)$ is an arbitrary function depending on $n$ only.

Finding $x$-integral, Part(a): Let us find an $x$-integral of equation (6.7) of minimal order if it exists. First, assume that equation (6.7) possesses an $x$-integral $F\left(x, n, t, t_{1}\right)$ of the first order. The equality 
$D_{x} F\left(x, n, t, t_{1}\right)=0$ can be rewritten as

$$
F_{x}+F_{t} t_{x}+F_{t_{1}} \frac{\left(1+M(n) t_{1}\right)\left(t_{1}-x\right)}{(1+M(n) t)(t-x)} t_{x}=0
$$

By comparing the coefficients before $t_{x}^{0}$ and $t_{x}$ we get

$$
F_{x}=0
$$

and

$$
F_{t}+F_{t_{1}} \frac{\left(1+M(n) t_{1}\right)\left(t_{1}-x\right)}{(1+M(n) t)(t-x)}+=0
$$

We differentiate equation (6.10) with respect to $x$, use (6.9), and get a contradictory equality

$$
\frac{\partial}{\partial x}\left\{\frac{t_{1}-x}{t-x}\right\}=0 .
$$

It means that equation (6.7) does not possess an $x$-integral $F\left(x, n, t, t_{1}\right)$ of the first order.

Now let us see whether equation (6.7) possesses an $x$-integral $F\left(x, n, t, t_{1}, t_{2}\right)$ of the second order. Since $D_{x} F=0$ then

$$
\begin{aligned}
& F_{x}+F_{t} t_{x}+F_{t_{1}} \frac{\left(1+M(n) t_{1}\right)\left(t_{1}-x\right)}{(1+M(n) t)(t-x)} t_{x} \\
& +F_{t_{2}} \frac{\left(1+M(n+1) t_{2}\right)\left(t_{2}-x\right)\left(1+M(n) t_{1}\right)\left(t_{1}-x\right)}{\left(1+M(n+1) t_{1}\right)\left(t_{1}-x\right)(1+M(n) t)(t-x)} t_{x}=0
\end{aligned}
$$

By comparing the coefficients before $t_{x}^{0}$ and $t_{x}$ we get

$$
F_{x}=0
$$

and

$$
F_{t}+F_{t_{1}} \frac{\left(1+M(n) t_{1}\right)\left(t_{1}-x\right)}{(1+M(n) t)(t-x)}+F_{t_{2}} \frac{\left(1+M(n+1) t_{2}\right)\left(t_{2}-x\right)\left(1+M(n) t_{1}\right)}{\left(1+M(n+1) t_{1}\right)(1+M(n) t)(t-x)}=0
$$

We differentiate equation (6.13) with respect to $x$ and get

$$
F_{t_{1}}\left(t_{1}-t\right)+F_{t_{2}} \frac{\left(1+M(n+1) t_{2}\right)\left(t_{2}-t\right)}{\left(1+M(n+1) t_{1}\right)}=0
$$

One can check that the system of partial differential equations (6.12), (6.13) and (6.14) is closed. To solve this system of equations we use the famous Jacobi Method: we first diagonalise the system (that is, we make it normal) and then we do the necessary changes of variables using the first integrals of the equations from the system. The calculations are standard but rather long. That is why we omit these straightforward steps and present an $x$-integral immediately. It is

$$
F\left(x, n, t, t_{1}, t_{2}\right)=\frac{\left(1+M(n+1) t_{2}\right)\left(t_{1}-t\right)}{(1+M(n) t)\left(t_{1}-t_{2}\right)}
$$

For the readers familiar with the characteristic rings (see [22], [8], [9]) we would like to note that the existence of a nontrivial $x$-integral for equation (6.7) implies that the characteristic ring $L_{x}$ 
in $x$-direction for this equation is of finite dimension. It is not difficult to see that for equation (6.7) characteristic ring $L_{x}$ is generated by three vector fields

$$
\begin{aligned}
& X_{1}=\frac{\partial}{\partial x} \\
& X_{2}=\frac{\partial}{\partial t}+\frac{\left(1+M(n) t_{1}\right)\left(t_{1}-x\right)}{(1+M(n) t)(t-x)} \frac{\partial}{\partial t_{1}}+\frac{\left(1+M(n+1) t_{2}\right)\left(t_{2}-x\right)\left(1+M(n) t_{1}\right)}{\left(1+M(n+1) t_{1}\right)(1+M(n) t)(t-x)} \frac{\partial}{\partial t_{2}}, \\
& X_{3}=\left(t_{1}-t\right) \frac{\partial}{\partial t_{1}}+\frac{\left(1+M(n+1) t_{2}\right)\left(t_{2}-t\right)}{\left(1+M(n+1) t_{1}\right)} \frac{\partial}{\partial t_{2}} .
\end{aligned}
$$

In partiacular, it means that the dimension of $L_{x}$ for equation (6.7) is 3.

Discretization, Part (b): Let us consider semi-discrete equations (1.1) possessing $n$-integral

$$
I=\frac{t_{x x}}{\sqrt{t_{x}}}+\frac{2 \sqrt{t_{x}}}{x+\varepsilon n}
$$

Since $D I=I$ then

$$
\frac{f_{x}+f_{t} t_{x}+f_{t_{1}} f+f_{t_{x}} t_{x x}}{\sqrt{f}}+\frac{2 \sqrt{f}}{x+\varepsilon(n+1)}=\frac{t_{x x}}{\sqrt{t_{x}}}+\frac{2 \sqrt{t_{x}}}{x+\varepsilon n}
$$

We compare the coefficients before $t_{x x}$ in (6.18) and get $f_{t_{x}} / \sqrt{f}=1 / \sqrt{t_{x}}$, or $\sqrt{f}=\sqrt{t_{x}}+L$, where $L$ is some function depending on $x, n, t, t_{1}$. We substitute $f=\left(\sqrt{t_{x}}+L\right)^{2}$ into (6.18) and have

$$
L_{x}+L_{t} t_{x}+L_{t_{1}}\left(\sqrt{t_{x}}+L\right)^{2}+\frac{\sqrt{t_{x}}+L}{x+\varepsilon(n+1)}=\frac{\sqrt{t_{x}}}{x+\varepsilon n}
$$

that implies that function $L\left(x, n, t, t_{1}\right)$ satisfies the following three differential equations

$$
\begin{gathered}
L_{t}+L_{t_{1}}=0 \\
2 L L_{t_{1}}+\frac{1}{x+\varepsilon(n+1)}=\frac{1}{x+\varepsilon n} \\
L_{x}+L^{2} L_{t_{1}}+\frac{L}{x+\varepsilon(n+1)}=0
\end{gathered}
$$

Equation (6.20) gives that

$$
L^{2}=\left(\frac{1}{x+\varepsilon n}-\frac{1}{x+\varepsilon(n+1)}\right) t_{1}+M
$$

where $M$ is some function depending on $x, n$ and $t$. We substitute the expression for $L^{2}$ from (6.22) into the equation (6.19) rewritten as $L L_{t}+L L_{t_{1}}=0$ and obtain

$$
M=\left(\frac{1}{x+\varepsilon(n+1)}-\frac{1}{x+\varepsilon n}\right) t+K
$$


where $K$ is some function depending on $x$ and $n$ only. Thus,

$$
L^{2}=\left(\frac{1}{x+\varepsilon n}-\frac{1}{x+\varepsilon(n+1)}\right)\left(t_{1}-t\right)+K
$$

Substitute this expression for $L^{2}$ into the equation (6.21) multiplied by $2 L$ and have

$$
K_{x}=\left(\frac{1}{x+\varepsilon(n+1)}-\frac{1}{x+\varepsilon n}\right) K \quad \rightarrow \quad K=\frac{C(n)}{(x+\varepsilon n)(x+\varepsilon(n+1))},
$$

where $C(n)$ is an arbitrary function of $n$. Therefore,

$$
L^{2}=\frac{\varepsilon\left(t_{1}-t\right)+C(n)}{(x+\varepsilon n)(x+\varepsilon(n+1))}
$$

and then

$$
f\left(x, n, t, t_{1}, t_{x}\right)=\left(\sqrt{t_{x}}+\sqrt{\frac{\varepsilon\left(t_{1}-t\right)+C(n)}{(x+\varepsilon n)(x+\varepsilon(n+1))}}\right)^{2}
$$

Let us note that one can eliminate function $C(n)$ in (6.23) by the change of variable $t_{(x, n)}=\tau(x, n)+$ $d(n)$, where $d(n)$ satisfies $\varepsilon(d(n+1)-d(n))+C(n)=0$. Equations possessing $n$-integral (6.17) become

$$
t_{1 x}=\left(\sqrt{t_{x}}+\sqrt{\frac{\varepsilon\left(t_{1}-t\right)}{(x+\varepsilon n)(x+\varepsilon(n+1))}}\right)^{2}
$$

Finding $x$-integral, Part (b): Let us find $x$-integral of equation (6.24). Denote by

$$
\alpha=\sqrt{\frac{\varepsilon\left(t_{1}-t\right)}{(x+\varepsilon n)(x+\varepsilon(n+1))}} \quad \beta=D \alpha=\sqrt{\frac{\varepsilon\left(t_{2}-t_{1}\right)}{(x+\varepsilon(n+1))(x+\varepsilon(n+2))}}
$$

We find an $x$-integral of the minimal order of equation (6.24) in the same way as we did for equation (6.7). We look for function $F\left(x, n, t, t_{1}, t_{2}\right)$ such that $D_{x} F=0$. We have,

$$
F_{x}+F_{t} t_{x}+F_{t_{1}}\left(t_{x}+\alpha^{2}+2 \sqrt{t_{x}} \alpha\right)+F_{t_{2}}\left(\sqrt{t_{x}}+\alpha+\beta\right)^{2}=0
$$

Compare the coefficients before $t_{x}, \sqrt{t_{x}}$ and $t_{x}^{0}$ in (6.26) and get the following system of equation

$$
\left\{\begin{array}{l}
F_{t}+F_{t_{1}}+F_{t_{2}}=0 \\
\alpha F_{t_{1}}+(\alpha+\beta) F_{t_{2}}=0 \\
F_{x}+\alpha^{2} F_{t_{1}}+(\alpha+\beta)^{2} F_{t_{2}}=0
\end{array}\right.
$$

that can be rewritten as

$$
\left\{\begin{array}{l}
F_{x}+\beta(\alpha+\beta) F_{t_{2}}=0 \\
\alpha F_{t}-\beta F_{t_{2}=0} \\
\alpha F_{t_{1}}+(\alpha+\beta) F_{t_{2}}=0
\end{array}\right.
$$

One can check that the system is closed and its solution is

$$
F=(x+\varepsilon n) \alpha-(x+\varepsilon(n+2)) \beta .
$$




\section{Continuum limits. Proof of Theorem 2.5}

Case $F$ : In semi-discrete equation (6.7) we rewrite $t(x, n)$ as $u(x, y), t_{1}$ as $u(x, y)+\varepsilon u_{y}(x, y), M(n)$ as $1 / R(\varepsilon n)=1 / R(y)$, and get

$$
u_{x}+\varepsilon u_{x y}=\left(\frac{R(y)+u+\varepsilon u_{y}}{R(y)+u}\right)\left(\frac{u+\varepsilon u_{y}-x}{u-x}\right) u_{x},
$$

or

$$
u_{x}+\varepsilon u_{x y}=\left(1+\frac{\varepsilon u_{y}}{u+R(y)}\right)\left(1+\frac{\varepsilon u_{y}}{u-x}\right) u_{x}
$$

or

$$
u_{x y}=u_{x} u_{y}\left(\frac{1}{u-x}+\frac{1}{u+R(y)}\right)+\varepsilon \frac{u_{y}^{2} u_{x}}{(u-x)(u+R(y))}
$$

Now we let $\varepsilon$ approach 0 to get continuous equation analogue

$$
u_{x y}=\left(\frac{1}{u-x}+\frac{1}{u+R(y)}\right) u_{x} u_{y} .
$$

Note that after the change of variable $\tilde{y}=-R(y)$ equation (7.1) becomes

$$
u_{x \tilde{y}}=\left(\frac{1}{u-x}+\frac{1}{u-\tilde{y}}\right) u_{x} u_{\tilde{y}}
$$

In $x$-integral $\varepsilon^{-1}\left(1+\left(1+n^{-1}\right) F\right)$ of semi-discrete equation (6.7), where $F$ is taken as (6.15) we substitute $u, u+\varepsilon u_{y}+(1 / 2) \varepsilon^{2} u_{y y}, 1 / R(y)$ and $y$ instead of $t, t_{1}, M(n)$ and $\varepsilon n$ correspondingly, and let $\varepsilon$ approach 0 to get its continuous analogue

$$
\tilde{F}=-\frac{u_{y y}}{u_{y}}+\frac{R^{\prime}(y)}{u+R(y)}+\frac{2 u_{y}}{u+R(y)}
$$

Note that continuous equation (7.1) possesses $y$-integral (6.1) and $x$-integral (7.3)

Case $G$ : In semi-discrete equation (6.24) we substitute $u, u+\varepsilon u_{y}$ and $y$ instead of $t, t_{1}$ and $\varepsilon n$ correspondingly, and let $\varepsilon$ approach 0 to get its continuous Liouville equation analogue

$$
u_{x y}=\frac{2 \sqrt{u_{x} u_{y}}}{x+y}
$$

In $x$-integral (6.27) multiplied by $-2 \varepsilon^{-2}$ we substitute $u, u+\varepsilon u_{y}+(1 / 2) \varepsilon^{2} u_{y y}$ and $y$ instead of $t, t_{1}$ and $\varepsilon n$ correspondingly, and let $\varepsilon$ approach 0 to get its continuous analogue

$$
\tilde{F}=\frac{u_{y y}}{\sqrt{u_{y}}}+\frac{2}{x+y}
$$

Note that continuous equation (7.4) possesses $y$-integral

$$
I=\frac{u_{x x}}{\sqrt{u_{x}}}+\frac{2 \sqrt{u_{x}}}{x+y}
$$

which is a continuous analogue of (6.17) and $x$-integral (7.5) 


\section{Discretization and the Bäcklund Transformation}

Recall the definition of the Bäcklund transformation for the PDE (see [16]). Suppose that $u(x, t)$ and $\tilde{u}(x, t)$ satisfy respectively differential equations

$$
E[u]=0
$$

and

$$
\tilde{E}[\tilde{u}]=0 .
$$

Here the expression $E[u]$ denotes the fact that $E$ depends on $u$ and a finite number of its derivatives. Then the set of the relations

$$
R_{j}[u, \tilde{u}]=0, \quad j=1,2, \ldots, k
$$

defines the Bäcklund transformation if these relations satisfy the following conditions: $\tilde{u}$ exists and solves (8.2) whenever $u$ exists and solves (8.1) and vice versa. When $u$ and $\tilde{u}$ are solutions of one and the equation then (8.3) defines the Bäcklund autotransformation. In that case we exclude the trivial autotransformation $u \equiv \tilde{u}$.

It is well-known that iterations of the Bäcklund autotransformation of a PDE define a semidiscrete equation. Semi-discrete models constructed in such a way are also called discretizations. Below we examine the question whether the semi-discrete equations found above by discretization preserving integrals do realize the Bäcklund autotransformation. The answer is stated in the following proposition.

Proposition 8.1. In cases $A, B, C, D, E, F$ from Theorem 2.5 the semi-discrete equations realize the Bäcklund autotransformations for their continuum limits, but in the case $G$ does not.

Scheme of the proof. For the case $B$ the proof is very simple. By differentiation of the equation

$$
u_{1 x}=u_{x}-e^{u}+e^{u_{1}}
$$

with respect to $y$ we find the equation

$$
u_{1 x y}-e^{u_{1}} u_{1 y}=u_{x y}-e^{u} u_{y}
$$

which is satisfied identically by means of the equation (II) from the Goursat list: $u_{x y}=e^{u} u_{y}$. Equation (8.5) immediately shows that all requests of the definition of the Bäcklund transformation are satisfied.

Concentrate on the case $A$ :

$$
u_{1 x}=u_{x}+C e^{\left(u_{1}+u\right) / 2}
$$

which is a discretization of the Liouville equation

$$
u_{x y}=e^{u}
$$

Differentiate (8.6) with respect to $y$ and get

$$
u_{1 x y}=e^{u}+(1 / 2) C e^{\left(u_{1}+u\right) / 2}\left(u_{1 y}+u_{y}\right)
$$


By differentiating (8.8) with respect to $x$ and simplifying by means of (8.6)-(8.8) we get

$$
u_{1 x x y}-u_{1 x y} u_{1 x}=0 .
$$

Reduce it to the convenient form $d\left(-u_{1}+\log u_{1 x y}\right) / d x=0$ and then integrate

$$
u_{1 x y}=C_{1}(y) e^{u_{1}} .
$$

Due to (8.10) equation (8.8) is rewritten as

$$
u_{1 y}=-u_{y}+C_{1}(y) e^{\left(u_{1}-u\right) / 2}-e^{\left(u-u_{1}\right) / 2} .
$$

Reasonings above result in the statement: relations (8.6), (8.11) define the Bäcklund transformation between equations (8.7) and (8.10). Choose $C_{1}(y)=1$ then this transformation becomes the Bäcklund auto-transformation for the Liouville equation which has been found by A.V.Bäcklund himself (see [11]).

Consider the case $G$. Let us prove that

$$
\sqrt{u_{1 x}}=\sqrt{u_{x}}+\sqrt{\frac{\varepsilon\left(u_{1}-u\right)}{(x+\varepsilon n)(x+\varepsilon(n+1))}}
$$

does not realize the Bäcklund autotransformation for the equation

$$
u_{x y}=\frac{2 \sqrt{u_{x}} \sqrt{u_{y}}}{x+y} .
$$

Assume contrary and differentiate (8.12) with respect to $y$. After simplification we get

$$
\sqrt{u_{1 y}}+\sqrt{u_{y}}=\frac{2 \sqrt{x+\varepsilon n} \sqrt{x+\varepsilon(n+1)}}{(x+y) \sqrt{\varepsilon}} \sqrt{u_{1}-u} .
$$

Now differentiate (8.14) with respect to $x$ and simplify by means of (8.12)-(8.14). As a result one gets a contradictory equation

$$
\frac{\sqrt{u_{1}-u}}{\sqrt{\varepsilon}}\left(\frac{\sqrt{x+\varepsilon(n+1)}}{\sqrt{x+\varepsilon n}}+\frac{\sqrt{x+\varepsilon n}}{\sqrt{x+\varepsilon(n+1)}}-2 \frac{\sqrt{x+\varepsilon n} \sqrt{x+\varepsilon(n+1)}}{x+y}\right)=0 .
$$

This proves that (8.12) does not realize the Bäcklund autotransformation for (8.13). Other statements of the Proposition 8.1 are proved in a similar way.

\section{Conclusion}

Darboux integrable equations or equations of Liouville type constitute a very well studied subclass of hyperbolic type PDE. The problem of complete description of this subclass was formulated and partly solved by E.Goursat in 1899 (see [6]). Since then many authors have investigated the classification problem (see [3], [12], [13], [18], [20] - [23]). To the best of our knowledge the problem up to now is still unsolved. The similar problem for the semi-discrete chains (1.1) and the fully discrete models is less studied. We can mention only particular classes of the equations investigated in [4], [8], [9], [20] and [21]. In the present article we discussed the problem of discretization via integrals and presented some new non-autonomous examples of the Darboux integrable chains. 


\section{Acknowledgments}

This work is partially supported by Russian Foundation for Basic Research (RFBR) grant 14-0197008-r-povolzhie-a.

\section{References}

[1] V. E. Adler, On a discrete analog of the Tzitzeica equation (arXiv:1103.5139).

[2] M. Bruschi, D. Levi, and O. Ragnisco, Discrete version of the nonlinear Schrdinger equation with linearlyx-dependent coefficients, Il Nuovo Cimento A Series 11 53(1) (1979) 21-30.

[3] N. F. Gareeva and A. V. Zhiber, The second order integrals of the hyperbolic equations and evolutionary equations, in Proceedings of the International Conference "Algebraic and analytic methods in the theory of the differential equations" (1996) Orel, edited by A.G.Meshkov, 39-42.

[4] R. N. Garifullin and R. I. Yamilov, Generalized symmetry classification of discrete equations of a class depending on twelve parameters, Journal of Physics A: Mathematical and Theoretical 45.34 (2012) 345205 .

[5] J. Gibbons and B. A. Kupershmidt, Time discretizations of lattice integrable systems, Physics Letters A $165.2(1992)$ 105-110.

[6] E. Goursat, Recherches sur quelques équations aux dérivés partielles du second ordre, Annales de la faculté des Sciences de l'Université de Toulouse 2e série, 1:1 (1899) 3178.

[7] I. T. Habibullin, N. Zheltukhina, and A. Sakieva, Discretization of hyperbolic type Darboux integrable equations preserving integrability, J. Math. Phys. 52 (2011) 093507.

[8] I. Habibullin I, N. Zheltukhina and A. Pekcan, On the classification of Darboux integrable chains, $J$. Math. Phys. 49 (2008) 102702.

[9] I. Habibullin, N. Zheltukhina and A. Pekcan, Complete list of Darboux integrable chains of the form $t_{1 x}=t_{x}+d\left(t, t_{1}\right), J$. Math. Phys. 50 (2009) 102710.

[10] R. Hirota and K. Kimura, Discretization of the Euler top, Journal of the Physical Society of Japan 69 (2000) 627-630.

[11] Nail H. Ibragimov, Transformation groups applied to mathematical physics, Vol. 3 Springer, 2001.

[12] O. V. Kaptsov, On the Goursat classification problem, Programming and Computer Software 38 (2) 102-104.

[13] M. E. Lainé, Sur une équation de la forme $s=p \phi(x ; y ; z ; q)$ integrable par la méthode de Darboux, Comptes rendus, 183 (1926) 1254-1256.

[14] J. Moser and A. P. Veselov, Discrete versions of some classical integrable systems and factorization of matrix polynomials, Communications in Mathematical Physics 139.2 (1991) 217-243.

[15] M. Murata, et al., How to discretize differential systems in a systematic way, Journal of Physics A: Mathematical and Theoretical 43.31 (2010) 315203.

[16] Alan C. Newell, Solitons in mathematics and physics, Philadelphia: Society for Industrial and applied Mathematics, 1985.

[17] Y. B. Suris, The problem of integrable discretization: Hamiltonian approach, 219 Springer, 2003.

[18] E. Vessiot, Sur les équations aux dérivés partielles du second order, $F(x ; y ; z ; p ; q ; r ; s ; t)=0$, integrable par la méthode de Darboux, J. Math. pure appl. 18 (1939) 1-61.

[19] D. Zakharov, A discrete analogue of the modified Novikov-Veselov hierarchy, (arXiv.org.nlin.arXiv:0904.3728v2).

[20] K. Zheltukhin and N. Zheltukhina, On existence of an $x$-integral for a semi-discrete chain of hyperbolic type, Journal of Physics Conference Series 670(1) (2016) 012055.

[21] K. Zheltukhin and N. Zheltukhina, Semi-discrete hyperbolic equations admitting five dimensional characteristic $x$-ring, Journal of Nonlinear Mathematical Physics 23(3) (2016) 351-367.

[22] A. V. Zhiber, R.D. Murtazina, I. T Habibullin and A. B. Shabat, Characteristic Lie rings and nonlinear integrable equation, M.Izhevsk (2012) 3-76 (in Russian).

[23] A. V. Zhiber and V. V. Sokolov, Exactly integrable hyperbolic equations of Liouville type, Russian Mathematical Surveys 56(1) (2001) 61-101 . 